\title{
Papel de los estimulantes de eritropoyesis en la anemia neonatal
}

\section{Role of erythropoiesis stimulants in neonatal anemia}

\author{
Mariana Roldan-Isaza ${ }^{1}$, Mabel Dahiana Roldan-Tabares ${ }^{1}$, Manuela Carvajal-Alzate ${ }^{1}$, \\ Natalia Morales-Quintero ${ }^{1}$, Gabriela Coronado-Magalhaes $^{1}$, Lina María Martínez-Sanchez ${ }^{2}$
}

\section{RESUMEN}

La anemia es una patología comúnmente encontrada en la población neonatal; es secundaria a una serie de factores de riesgo fisiológicos y no fisiológicos que contribuyen a su desarrollo. Entre sus variantes se encuentra la enfermedad hemolítica del feto y el recién

\begin{abstract}
Anemia is a pathology commonly found in the neonatal population, due to a series of physiological and non-physiological risk factors that contribute to the development of the disease. Among the variants is hemolytic disease of the fetus and newborn, which
\end{abstract}

\section{Historial del artículo:}

Fecha de recepción: 26/10/2020

Fecha de aprobación: 15/04/2021

1 Universidad Pontificia Bolivariana, Escuela de Ciencias de la Salud, Facultad de Medicina, Estudiante de Medicina. Medellín, Colombia.

2 Universidad Pontificia Bolivariana, Escuela de Ciencias de la Salud, Facultad de Medicina, Bacterióloga, Especialista en Hematología, Magister en Educación. Medellín, Colombia.

Correspondencia: Mariana Roldan Isaza. Universidad Pontificia Bolivariana, Calle 78BN - 72 A 109, Medellín, Colombia. Teléfono: +57(4) 4488388. Correo electrónico: mariana.roldan@upb.edu.co

Como citar este artículo: Roldan-Isaza M, Roldan-Tabares MD, Carvajal-Alzate M, Morales-Quintero N, Coronado-Magalhaes G, Martínez-Sanchez LM. Papel de los estimulantes de eritropoyesis en la anemia neonatal. Revista de la Facultad de Ciencias de la Salud de la Universidad del Cauca. 2021;23(2):17-26. https://doi.org/10.47373/rfcs.2021.v232.1672 
nacido, la cual es considerada la causa más frecuente de anemia en dicha población. Se realizó la revisión bibliográfica en las bases de datos Medline, Science Direct y Lilacs hasta agosto de 2020. En la búsqueda y selección de los artículos se usaron los términos MeSH: anemia neonatal, eritroblastosis fetal y eritropoyetina. Para el tratamiento de dicha patología se dispone de la transfusión de glóbulos rojos y los agentes estimulantes de la eritropoyesis; el uso de eritropoyetina humana recombinante como parte del tratamiento ha sido ampliamente discutido y controvertido, no obstante, hay estudios que demuestran que su uso reduce la necesidad de transfusiones sanguineas.

Palabras clave: Anemia Neonatal; Eritroblastosis Fetal; Eritropoyetina; Recién Nacido; Isoinmunización Rh.

\section{INTRODUCCIÓN}

Anemia neonatal es un problema comúnmente encontrado en recién nacidos en unidades de cuidados intensivos neonatales (1). Esta puede ser definida como la disminución de la concentración de hemoglobina o del hematocrito por debajo de dos desviaciones estándar con respecto a la media correspondiente a su edad y sexo $(1,2)$; también puede ser definida como una media de hemoglobina $16.9 \pm 1.6 \mathrm{~g} / \mathrm{dL}$ en recién nacido a término y de $15.9 \pm 2.4 \mathrm{~g} / \mathrm{dL}$ en pretérminos (3).

En el periodo neonatal las principales causas de anemia se pueden atribuir a pérdidas sanguíneas, hemólisis y menor producción de glóbulos rojos, siendo la hemólisis la principal causa (4). Entre las generadas por pérdidas sanguíneas se encuentran placenta previa, transfusión fetomaterna, hemorragia intracraneal o cefalohematoma, o causas iatrogénicas tales como extracciones múltiples de muestras de sangre para estudios (5). Las causas hemolíticas originan anemias de tipo regenerativas, se presentan por causas intrínsecas y extrínsecas, conocidas como anemias hemolíticas corpusculares y extra corpusculares, respectivamente (6).

La anemia hemolítica secundaria a incompatibilidad de grupo sanguíneo materno fetal o también conocida como aloinmunización de glóbulos rojos maternos, es la más frecuente en este grupo poblacional (7). Se produce por una reacción inmunitaria en la madre que conlleva a la formación de anticuerpos contra los antígenos de los glóbulos rojos fetales, permitiendo así su hemólisis, se is considered the most frequent cause of anemia in this population. The bibliographic review was carried out in databases such as Medline, Science Direct and Lilacs until august 2020. The search and selection of the articles was done through the presence of the following MeSH terms: Anemia, Neonatal; Erythroblastosis, Fetal; Erythropoietin. Treatmentofanemiaincludestransfusion of red blood cells and erythropoiesis-stimulating agents; the use of recombinant human erythropoietin as part of treatment has been widely discussed and controversial. However, there are studies that show that its use reduces the need for blood transfusions.

Key words: Anemia, Neonatal; Erythroblastosis, Fetal; Erythropoietin; Infant, Newborn, Rh Isoimmunization.

reporta que 35 por cada 10.000 nacidos vivos están en riesgo de desarrollar enfermedad hemolítica del feto y el recién nacido (EHFRN) debido a la aloinmunización $(8,9)$.

Otra causa común de anemia en esta población es la prematuridad, el muy bajo peso al nacer (menor a 1500 gr) y el de peso extremadamente bajo al nacer (menor de 1000 gr). Esto se debe a que posterior al nacimiento, los niveles de hemoglobina sufren un descenso fisiológico, siendo más precoz y marcado en los neonatos pretérmino que en los a término (10). Esta reducción se presenta principalmente debido a una falta de respuesta por parte de la eritropoyetina (EPO) frente a la anemia generada, aunque también contribuye las extracciones de sangre repetidas, la reducción de la vida media del eritrocito y el crecimiento rápido del neonato (11).

Algunas de las manifestaciones clínicas en los neonatos con anemia son la palidez de piel y mucosas, e ictericia. Cuando la anemia es aguda aparecen signos de hipovolemia y choque tales como taquicardia, taquipnea, hipotensión, mala perfusión y acidosis metabólica. En las anemias hemorrágicas crónicas, predomina la palidez con poca o nula sintomatología respiratoria y hemodinámica. Las anemias hemolíticas se caracterizan por presentar ictericia, palidez y hepatoesplenomegalia; pueden generar complicaciones a corto y largo plazo como falla cardiaca, edema, ascitis, alteración en el desarrollo cerebral debido a la acumulación de bilirrubina libre no conjugada y liposoluble en el tejido nervioso, entre otras, incluso la muerte neonatal $(6,12)$. 
El tratamiento de la anemia en el neonato incluye la transfusión de glóbulos rojos y el uso de agentes estimulantes de la eritropoyesis; la primera siendo una de las estrategias más efectivas para una rápida reposición de volumen eritrocitario, pero que tiene algunos riesgos tales como la sensibilización inmunológica e infecciones virales (10), incluso un aumento en la frecuencia de administración, puede estar asociado con displasia broncopulmonar y retinopatía del prematuro (11).

La administración de agentes estimulantes de la eritropoyesis llega como una adecuada estrategia en estudio para el tratamiento de estos pacientes (13) y ha tomado gran importancia en los recién nacidos pretérmino. Esta revisión narrativa presenta los aspectos generales de la anemia neonatal enfocado en el papel de los agentes estimulantes de eritropoyesis como tratamiento de esta entidad. Se realizó una revisión bibliográfica en las bases de datos Medline, Science Direct y Lilacs hasta agosto de 2020. La búsqueda y selección de los artículos se realizó usando los términos MeSH: Anemia, Neonatal; Erythroblastosis, Fetal; Erythropoietin.

\section{EPIDEMIOLOGÍA}

Para el ańo 2010 la anemia representó aproximadamente 64.8 millones de ańos de vida con discapacidad y el $9 \%$ de la carga global total de discapacidad para todas las condiciones. La anemia repercute de forma negativa en los nacimientos prematuros, bajo peso al nacer, retraso en el desarrollo y mortalidad perinatal (14).

Dentro de las entidades que promueven la hemolisis de los glóbulos rojos, se encuentra la EHRN, la cual se presenta con mayor frecuencia en personas blancas con un $15 \%$, seguido de personas hispanas con un $8 \%$ (15). Antes de implementarse la inmunoprofilaxis como tratamiento preventivo de la EHFRN, la incidencia era del 16\% y la mortalidad atribuida a esta patología se presentaba en más de un $50 \%$ de los recién nacidos. Actualmente, su incidencia es del $2 \%$ con una mortalidad asociada del $16 \%(16,17)$.

La causa más frecuente de EHFRN está dada por anticuerpos Rh, cuya incidencia en la población caucásica está por debajo del $0.2 \%$, debido al uso de la inmunoglobulina anti-D en maternas con Rh negativo como estrategia profiláctica. Sin embargo, en algunos países en desarrollo, los programas para la detección de maternas con riesgo de desarrollar aloinmunización y el uso de tratamiento profiláctico no están bien establecidos, por lo cual la EHFRN sigue siendo una causa importante de morbimortalidad en el neonato (18).
A pesar de una buena inmunoprofilaxis, 1 a 2 de cada 1000 maternas van a desarrollar anticuerpos anti-D (16). La mortalidad en pacientes con EHFRN depende de la severidad de la enfermedad en el neonato, las afectaciones leves a moderadas tienen una baja mortalidad y son las presentaciones más frecuentes en el $50 \%$ de los pacientes. Si la afectación es severa la mortalidad puede ser de un $30 \%$ (17).

Las causas de anemia hipoplásica en el recién nacido son múltiples, dentro de este grupo la entidad más frecuente es la anemia fisiológica del recién nacido. Esta se presenta en prematuros y recién nacidos con un peso menor a los $1500 \mathrm{gr}$; su incidencia se relaciona con la prematurez y el bajo peso al nacer (10). Otra de las entidades de anemia hipoplásica son las secundarias a infecciones; dentro de este grupo se encuentra la infección congénita por rubéola, cuya incidencia ha disminuido desde la vacunación (19). Por último, está la anemia aplásica congénita, entidad poco común que afecta a los recién nacidos, su incidencia varía de acuerdo con la región geográfica (20).

Actualmente se estima que 38.000 neonatos prematuros reciben al ańo más de 300.000 transfusiones para el tratamiento de anemia. Desde los ańos noventa se incorporó la administración de eritropoyetina recombinante humana (rHu-EPO) en el tratamiento de anemia en los prematuros. Esta molécula ha reducido favorablemente la necesidad de transfusiones de glóbulos rojos y el volumen de estas. Otros autores concluyeron que los neonatos muy prematuros y con estado clínico grave son propensos a más extracciones de muestras sanguíneas y, por lo tanto, requieren más transfusiones; pero al aplicar rHu-EPO, se redujo en un $40 \%$ la necesidad de estas, teniendo un mayor impacto en prematuros con peso entre 1000 y 1249 gr (10).

EnColombia,ennińosmenoresde2 ańoshayunaprevalencia de anemia por déficit de hierro de aproximadamente 53.2\% siendo las zonas rurales las más afectadas (21). Por otro lado, en la anemia falciforme, se estimó una cifra anual de nacidos vivos con rasgo drepanocítico (heterocigótico) de aproximadamente 20.000 nińos versus 500 nińos nacidos siendo homocigotos para la $\mathrm{HbS}$. Hasta el momento no se encuentran cifras oficiales que definan una real frecuencia de la anemia neonatal en Colombia (22).

\section{FISIOPATOLOGÍA}

Durante el periodo neonatal el recién nacido debe realizar la transición entre la vida uterina a la vida extrauterina mediante múltiples fenómenos de adaptación fisiológica 
con el fin de alcanzar la independencia de la madre necesaria para una vida extrauterina sana. Estos fenómenos de adaptación producen alteraciones en los parámetros hematológicos normales desde el momento del nacimiento y durante toda la etapa neonatal, planteando retos en su diagnóstico y tratamiento $(23,24)$.

Existen diversos parámetros hematológicos de laboratorio que se presentan en la anemia del recién nacido tales como el número de glóbulos rojos, el hematocrito o la concentración de hemoglobina $>2$ desviaciones estándar por debajo de la media específica para la edad correspondiente para cada paciente (25).

La hemólisis patológica que se presenta en el periodo neonatal por la destrucción prematura y acelerada de los glóbulos rojos sigue siendo la principal causa de anemias regenerativas en el período neonatal. Como origen de esta hiper hemólisis pueden existir causas extrínsecas a los glóbulos rojos, conocidas como anemias hemolíticas extracorpusculares que a su vez pueden ser de origen inmunológico (incompatibilidad $\mathrm{ABO}$ o $\mathrm{Rh}$ ), infeccioso (paludismo, rubéola, toxoplasmosis) o mecánico (hemangioma, síndrome hemolítico urémico) o bien causas intrínsecas clasificadas como anemias hemolíticas corpusculares (enfermedades de la membrana eritrocítica o hemoglobinopatías) (6).

Las anemias hemolíticas extracorpusculares son las más frecuentes en el recién nacido y algunas veces pueden necesitar tratamiento urgente (26); entre ellas está las causas inmunológicas como la incompatibilidad ABO que se da en recién nacidos de madre de grupo sanguíneo 0 con anticuerpos anti-A y anti-B que destruyen los hematíes portadores de los neonatos de grupos A o B. Otra de las causas inmunológicas es la isoinmunización Rh D. Desde la gestación las pacientes con el grupo sanguíneo Rh negativo (Rh d) que de alguna forma estuvieron expuestas o en contacto con el grupo sanguíneo $R h$ positivo (Rh D) corren riesgo de desarrollar anticuerpos anti-D (Anti-Rhd) por la sensibilización de los glóbulos rojos maternos cuando estos se exponen a antígenos eritrocitarios durante el embarazo, es decir un feto Rh D-positivo o antígenos no propios derivados de alguna transfusión que se haya realizado durante la vida $(27,28)$.

La anemia por pérdida de sangre a su vez se divide en: antes o durante el parto o en el periodo neonatal (1). Las hemorragias antes o durante el parto es la hemorragia fetal que generalmente se dan por fisiopatológicas tales como la transfusión fetomaterna, fetoplacentaria y la etiología feto-fetal en donde en un embarazo gemelar la placenta es monocorial y con anastomosis arteriovenosas que produce la transfusión de feto a otro y iatrógenica (5). Las que ocurren durante el parto, pueden darse por diversas causas como la hemorragia placentaria por mecanismos como la placenta previa o el desprendimiento de placenta; la hemorragia del cordón umbilical por vasos aberrantes, la inserción velamentosa o el hematoma del cordón (29).

Por otro lado, la anemia hemorrágica en el periodo neonatal puede presentarse como consecuencia de una coagulopatía adquirida debido a la deficiencia de los factores de coagulación dependientes de la vitamina K (II, VII, IX y X) (5). También puede darse debido a una hemorragia intracraneal que ocurre en la prematuridad, en el segundo gemelo, en los partos podálicos o partos precipitados y en hipoxia $(5,30)$. Otras causas de sangrado neonatal son dadas por mecanismos como un cefalohematoma masivo, la hemorragia subgaleal o el caput hemorrágico; por sangrados retroperitoneales provenientes del rinón o la glándula suprarrenal, por ruptura esplénica o hepática; por sangrado gastrointestinal, cuyos desencadenantes típicamente son la enterocolitis necrosante (30-32). También pueden ocurrir sangrados neonatales por iatrogenia (1).

En cuanto a la anemia hipoplásica se describe que puede ser fisiológica en los recién nacidos a término alrededor de la sexta o doceava semana de vida, y en el caso de los recién nacidos prematuros a partir de la cuarta hasta la décima semana (5). Otras etiologías de la anemia hipoplásica en el recién nacido son la anemia aplásica congénita (anemia de Blackfand-Diamond, anemia de Fanconi, diseritropoyética, Estren-Damesheck, aplásica idiopática) y la anemia aplásica secundaria (leucemia congénita, infecciones como rubéola, parvovirus B19, AlbersSchonberg, Benjamin, anemia postransfusión ya sea extrauterina o intrauterina por isoinmunización) (5).

\section{ADMINISTRACIÓN TEMPRANA DE AGENTES ESTIMULANTES DE LA ERITROPOYESIS}

Desde finales de la década de 1980, el gen de la eritropoyetina (EPO) humana fue clonado y la eritropoyetina humana recombinante (rHu-EPO) y otros agentes estimulantes de la eritropoyesis estuvieron disponibles, como agentes para la farmacoterapia $(33,34)$. El tratamiento con rHu-EPO ha abierto una nueva perspectiva en el tratamiento de la anemia en recién nacidos aun cuando su indicación, dosis y momento de inicio no se encuentran claramente establecidos (35). 
En diferentes estudios se ha visto que los recién nacidos que reciben dosis adecuadas y oportunas de EPO (o darbepoetina) requieren menos transfusiones y a su vez reduce la exposición a más donantes (11). También se reducen sus efectos adversos como las reacciones transfusionales agudas (en las primeras 24 horas posteriores a la transfusión) o tardías (entre 4 y 14 días posttransfusión). La más frecuente es la reacción febril inducida por citoquinas, la isoinmunizacion contra el antígeno HLA y otras menos frecuentes como la incompatibilidad ABO, la injuria pulmonar aguda y la infección bacteriana $(36,37)$.

El porcentaje de glóbulos rojos circulantes disminuye después del nacimiento en todos los lactantes, particularmente en los recién nacidos prematuros a consecuencia de una baja respuesta a la anemia. Los niveles plasmáticos de EPO son bajos, lo que justifica fisiopatológicamente el uso de agentes estimulantes de la EPO para prevenir o tratar la anemia $(10,38)$.

La EPO es una hormona glucoprotéica, producida y regulada principalmente a nivel renal, que ayuda en la regulación de la eritropoyesis; esta se une al receptor de la EPO que se encuentra en las células progenitoras eritroides dentro de la médula ósea, provocando la producción de eritrocitos (39). Además de estimular la eritropoyesis, inhibe la apoptosis en células vasculares y neuronas y regula la angiogénesis; su expresión depende de la presión arterial de oxígeno y está regulada por la hipoxia $(40,41)$. El uso de eritropoyetina humana recombinante (rHu-EPO) como parte del tratamiento de la anemia del prematuro ha sido ampliamente discutido y controvertido, ya que la administración precoz de esta no reduce el número de transfusiones recibidas $(5,41,42)$; sin embargo, hay estudios que demuestran una disminución en la necesidad de transfusiones sanguíneas $(13,43,44)$.

Las indicaciones actuales para la administración de EPO se limitan a neonatos prematuros con un peso al nacer entre 1000 gr a $1250 \mathrm{gr}$, acompańado de algún otro factor de riesgo para requerir transfusión, necesidad de ventilación con presión positiva continua durante más de 48 horas o extracciones sanguíneas en las primeras 48 horas $(34,40)$. A menor edad gestacional y peso al nacimiento, mayor es la necesidad de reposición rápida de hematíes, siendo los principales factores predictivos relacionados con la necesidad de transfusión e inicio de tratamiento con rHu-EPO (10).

Existen dos formas de administración de los estimulantes de la eritropoyesis: temprana, antes de 8 días de edad cronológica, y la tardía entre los 8 y 28 días de vida. Cada uno de estos ha sido asociado a algunos beneficios, sobre todo en pretérminos y con bajo peso al nacer (menor de 2500 gr) (11,38). Con la administración temprana se ha encontrado una reducción del uso de transfusiones de glóbulos rojos, el volumen recibido de estas células y la exposición a nuevos donantes; sin diferencias en la retinopatía del prematuro (ROP) y la mortalidad con la administración temprana de estos estimulantes, pero con disminución significativa de la incidencia de hemorragia intraventricular (HIV), leucomalacia periventricular (LPV) y enterocolitis necrozante (ECN) (11).

Con la administración tardía de los estimulantes también se encontró una reducción en el uso de transfusiones de glóbulos rojos, pero no en el total del volumen administrado de glóbulos rojos; a diferencia de la administración temprana, se vio un incremento del riesgo de ROP sin diferencias en relación con mortalidad y ECN (38).

El tratamiento de la anemia incluye transfusión de glóbulos rojos y el uso de agentes estimulantes de la eritropoyesis. La transfusión tiene un efecto inmediato en la corrección de la anemia del prematuro, principalmente en un paciente que requiere una reposición rápida del volumen eritrocitario en los primeros días de vida. Sin embargo, existen riesgos como la sensibilización inmunológica y la transmisión de infecciones virales (10). Por esta razón el uso de agentes estimulantes de la eritropoyesis se ha convertido en un blanco importante de estudio, volviendo urgente la necesidad de enfocar las guías de tratamiento en el uso de estos y de esta manera disminuir los riesgos existentes al recibir una transfusión sanguínea (13).

Existen distintos tipos de eritropoyetina; la primera desarrollada como tratamiento fue la eritropoyetina recombinante conocida como epoetina alfa y beta creada en 1977 y tiene la misma estructura que la eritropoyetina natural del organismo, luego en el 2002 aparece la darpoetina alfa un análogo hiperglicosilado de la EPO que estimula la eritropoyesis por el mismo mecanismo que la hormona endógena, con una vida media superior a la epoetina alfa y beta.

La darpoetina actualmente es la más descrita en los estudios como tratamiento de anemia en neonatos con hallazgos de reducción en el número de transfusiones requeridas (11); esta tiene la ventaja con respecto a la $\mathrm{EPO}$ que se puede administrar con menor frecuencia debido a que es un estimulante de larga duración. En el 2007 surge la metoxi-polietilenglicol epoetina beta que activa constantemente el receptor de la eritropoyetina aumentando así la semivida (39). 
La darbepoietina alfa tiene una vida media prolongada permitiendo el uso de una sola dosis a la semana en contraste con la rHu-EPO que requiere tres dosis por semana. En un estudio realizado por Ohls et al. donde se evaluó la efectividad de la rHu-EPO y darbepoetina alfa en recién nacidos pretérmino, se encontró que tanto los pacientes que utilizaban rhEPO como darbepoietina requerían menos transfusiones. Ambos medicamentos se pueden considerar equiparables a la hora de escoger uno u otro para el tratamiento de la anemia; sin embargo, la darbepoietina requiere menos dosis que la rhEPO (13).

Un estudio para determinar el efecto de rHu-EPO en la prevención de anemia tardía en neonatos con isoinmunización Rh que habían sido transfundidos in utero, usó protocolos de tratamiento de rHU-EPO administrada a los neonatos a dosis de $200 \mathrm{U} / \mathrm{Kg} /$ día y $400 \mathrm{U} / \mathrm{Kg} /$ día. En estos estudios no evidenció diferencias en el efecto de la rHu-EPO en ambos grupos indicando que no es necesario el incremento de la dosis para prevenir la anemia tardía; además, se observó una reducción en la necesidad de terapia de transfusión con plasma fresco congelado y ninguno de los pacientes presentó efectos secundarios (43). Esto último contrasta con otros estudios donde la terapia de rHu-EPO parece aumentar el riesgo de desarrollo y deterioro de la ROP (45).

En la última década, se ha estudiado el beneficio que puede tener la rhEPO y darbepoetina en la neuroprotección neonatal (46). Este es un tema que requiere más estudios.

\section{FACTORES DE RIESGO}

En los neonatos nacidos a término, es común que ocurra una anemia fisiológica durante los primeros días o semanas de la vida postnatal, secundaria a la caída de la hemoglobina que dura aproximadamente hasta la semana 10 donde empieza a subir nuevamente de forma gradual (47). Sin embargo, en los prematuros y en aquellos con bajo peso al nacer, esta anemia fisiológica tiene unos valores significativamente más bajos en comparación con en los nacidos a término $(47,24)$. Otras condiciones patológicas que son clínicamente importantes en esta patología en los recién nacidos prematuros incluyen hemorragias, infecciones, ingesta inadecuada de nutrientes y enfermedades cardiorrespiratorias (48).

Otro factor de riesgo es el manejo de los vasos placentarios en el momento del nacimiento, puesto que estos contienen entre $1 / 3$ y $1 / 4$ del volumen sanguíneo del neonato. El volumen sanguíneo que es transferido del cordón umbilical al recién nacido es directamente proporcional al tiempo que se mantenga unido al cordón una vez se da el nacimiento; por lo tanto, una sujeción corta al cordón umbilical (teniendo en cuenta que en un minuto se realiza la mitad de la transfusión placentaria) es un factor de riesgo para anemia en el neonato (49).

El tipo de nacimiento también se ha relacionado con hematocrito más bajo en el período neonatal inmediato. Durante el nacimiento, las arterias umbilicales se contraen para mantener el aporte sanguíneo, sin embargo, la vena umbilical se mantiene dilatada y es la posición del neonato respecto a la placenta lo que mantiene el flujo de la madre a este. Cuando el recién nacido se encuentra por encima de la placenta (como ocurre, por ejemplo, en una cesárea) hay un flujo retrógrado hacia la madre, lo cual es un factor de riesgo para anemia (49). Lubetzky et al, compararon el hematocrito en recién nacidos a término sanos, que nacieron en cesárea electiva versus los nacidos por parto vaginal y encontraron que el hematocrito fue significativamente inferior en los neonatos nacidos por cesárea $(50,51)$.

Por otro lado, algunos factores como antecedentes de sangrado vaginal, placenta previa, compresión del cordón umbilical, cesárea y desprendimiento prematuro de la placenta, influyen negativamente en el volumen sanguíneo del neonato, convirtiéndose en factores de riesgo para el desarrollo de anemia en el recién nacido (52). Otro factor de riesgo es el embarazo gemelar. Se ha reportado una prevalencia de anemia en uno de los recién nacidos hasta en un $4.7 \%$ de los embarazos gemelares, la cual aumenta hasta $15 \%$ cuando son monocoriónicos $(53,54)$. En la Tabla 1 se resumen las causas y factores de riesgo para desarrollar anemia durante el periodo neonatal $(24,55)$.

Además de los factores de riesgo fisiológicos, la pérdida de sangre que acompańa las pruebas de laboratorio frecuentes es un factor no fisiológico que contribuye al desarrollo de anemia durante el período neonatal (56).

\section{COMPLICACIONES}

La anemia neonatal aguda puede llevar a condiciones que amenazan la integridad y la vida del recién nacido debido a que en sus presentaciones de mayor severidad puede causar choque hipovolémico e hipoxia al nacer (56). Cuando hay una disminución en los niveles de hemoglobina, se genera un déficit en el transporte de oxígeno a los tejidos e hipoxia que aumenta la disfunción orgánica en el neonato y en consecuencia se produce insuficiencia cardiaca, 
Tabla 1: Causas y factores de riesgo para anemia neonatal

Pérdida de sangre

Antes y durante el parto

- Hemorragia placentaria (placenta previa, desprendimiento de placenta)

- Hemorragia de cordón umbilical (vasos aberrantes, inserción velamentosa, hematoma de cordón)

- Hemorragia fetal: transfusión fetomaterna ( $8 \%$ de los embarazos, crónica o aguda), fetoplacentaria (cesárea con extracción del feto por encima del nivel de la placenta, tumoración placentaria, hematoma, nudos o prolapso oculto de cordón), feto fetal (placenta monocorial con anastomosis arteriovenosas), iatrógena

\section{Período neonatal}

- Enfermedad hemorrágica del recién nacido

- Intracraneal: en relación con prematuridad, segundo gemelo, parto de nalgas o parto rápido, hipoxia

- Cefalohematoma masivo, hemorragia subgaleal o caput hemorrágico

- Retroperitoneal: renal o suprarrenal

- Rotura hepática o esplénica

- Gastrointestinal: ulcus, enterocolitis necrosante, sonda nasogástrica (descartar deglución de sangre materna)

- Umbilical

- Anemia iatrógena (extracciones múltiples, sobre todo en el prematuro)

\section{Aumento de la destrucción de hematíes}

- Anemia hemolítica inmune (incompatibilidad grupo ABO y Rh, enfermedad autoinmune materna, lupus eritematoso sistémico, anemia hemolítica autoinmune, inducida por fármacos como ácido valproico, penicilina)

- Alteraciones enzimáticas (déficit glucosa-6-fosfato DH, déficit de piruvatocinasa)

- Defectos en la membrana de los hematíes (esferocitosis, eliptocitosis)

- Hemoglobinopatías (síndromes talasémicos)

- Infecciones (sepsis bacterianas o víricas, infecciones congénitas [TORCH])

- Alteraciones mecánicas de hematíes (CID, hemangiomas)

- Carencia de vitamina E

- Metabolopatías (galactosemia, osteopetrosis)

\section{Anemia hipoplásica}

- Anemia hipoplásica fisiológica: en el RN a término (6-12 semanas de vida), en el RN prematuro (4-10 semanas de vida)

- Anemia aplásica congénita: anemia de Blackfand-Diamond, anemia de Fanconi, diseritropoyética, Estren-Damesheck, aplasia idiopática

- Anemia aplásica secundaria: leucemia congénita, infecciones (rubéola, parvovirus B19), AlbersSchonberg, Benjamin, anemia postransfusión (extrauterina o intrauterina por isoinmunización) 
enfermedades inflamatorias, deterioro cognitivo, retardo motor y aumento de la mortalidad (57).

Entre las complicaciones de la anemia neonatal se encuentran la hemorragia intraventricular, la leucomalacia periventricular, la displasia broncopulmonar, la encefalopatía hipóxicoisquémica y la muerte $(58,59)$. También es un factor de riesgo independiente para mortalidad y la probabilidad de recibir una transfusión de sangre en prematuros nacidos con $<32$ semanas de gestación $(1,60)$. La anemia a largo plazo tiene el potencial de afectar el crecimiento cerebral tanto en prematuros como nacidos a término $(1,61,62)$. En los neonatos prematuros, después de la prematurez extrema y la hemorragia intracraneana, la anemia es el factor que más incide en la morbilidad neurológica (62).

\section{CONCLUSIONES}

La anemia neonatal es una entidad que el profesional de la salud debe sospechar, diagnosticar, tratar para evitar las posibles complicaciones derivadas. El tratamiento de la anemia en el recién nacido incluye la transfusión de glóbulos rojos y el uso de agentes estimulantes de la eritropoyesis; sin embargo, la terapia transfusional puede traer consigo algunos desenlaces adversos, por lo que la administración de agentes estimulantes de la eritropoyesis solos o como terapia complementaria a la transfusión debe ser considerada. La rHu-EPO y darbepoetina pueden tener beneficios en la neuroprotección neonatal, aunque se requieren más estudios que respalden estos hallazgos.

\section{CONTRIBUCIÓN DE LOS AUTORES}

Todos los autores colaboraron en la búsqueda y revisión de artículos, redacción, edición y corrección del documento final.

\section{CONFLICTOS DE INTERÉS}

No se declara ningún conflicto

\section{FUENTES DE FINANCIAMIENTO}

Ninguna

\section{REFERENCIAS}

1. Colombatti R, Sainati L, Travisanuto D. Anemia and transfusion in the neonate. Semin fetal neonat $\mathrm{m}$. 2016;21(1): 2-9 Doi: 10.1016/j.siny.2015.12.001

2. Pavo García M. R, Muńoz Díaz M., Baro Fernández M. Anemia en la edad pediátrica. Form Act Pediatr Aten Prim. 2016;9(4):149-55

3. Mamoury, G., Hamedy, A., Akhlaghi, F. Cord Hemoglobin in Newborns in Correlation with Maternal Hemoglobin in Northeastern Iran. Iranian Journal of Medical Sciences, 2015; 28(4): 166-168

4. Peńa A, Urrutia ML, Vera GH. Anemia neonatal por transfusión feto-materna: reporte de un caso. Rev Mex Pediatr. 2014;81(6):218-221

5. Bonastre-Blanco E, Thió-Lluch M, Monfort-Carretero L. Anemia neonatal. An. de Pediatria Contin. 2010; 8(2): 73-80. Doi: 10.1016/S1696-2818(10)70013-5

6. Da Costa L. Anémies hémolytiques du nouveau-né. EMC (Elsevier Masson SAS, Paris), Pédiatrie, 4-002-R-40, 011

7. Provan D, Baglin T, Dokal I. Vos J. Hematología pediátrica. En: Pérez G. Manual de hematología clínica. Vol 4. Espańa: Elsevier. 2017; p. 559-634

8. Li L, Huang L, Luo G, Luo Y, Fang Q. Prenatal treatment of severe fetal hemolytic disease due to anti-M alloimmunization by serial intrauterine transfusions. Taiwan J Obstet Gynecol. 2017; 56(3):379-381. Doi:10.1016/j.tjog.2017.04.022

9. Markham KB, Rossi KQ, Nagaraja HN, O'Shaughnessy RW. Hemolytic disease of the fetus and newborn due to multiple maternal antibodies. Am J Obstet Gynecol. 2015;213(1):68.e1-68.e5. Doi:10.1016/j. ajog.2015.01.049

10. López-Catzín JF, Bolado-García PB, Gamboa-López GJ, et al. Disminución de transfusiones en prematuros con anemia tratados con Eritropoyetina. Rev Med Inst Mex Seguro Soc. 2016;54(5):576-580

11. Ohlsson A, Aher SM. Early erythropoiesis-stimulating agents in preterm or low birth weight infants. Cochrane Database Syst Rev. 2017 Nov 16;11(11):CD004863. Doi: 10.1002/14651858.CD004863.pub5

12. Delaney M, Matthews DC. Hemolytic disease of the fetus and newborn: managing the mother, fetus, and newborn. Hematology Am Soc Hematol Educ Program. 2015; 2015:146-151. Doi:10.1182/ asheducation-2015.1.146

13. Ohls RK, Christensen RD, Kamath-Rayne BD, Rosenberg A, Wiedmeier SE, Roohi M, et al. A Randomized, Masked, Placebo Controlled Study of Darbepoetin Alfa in Preterm Infants. PEDIATRICS 2013; 132(1): e119-e127 
14. Chaparro CM, Suchdev PS. Anemia epidemiology, pathophysiology, and etiology in low- and middleincome countries. Ann N Y Acad Sci. 2019; 1450(1): 15-31. Doi:10.1111/nyas.14092

15. Ifeanyi OE. Hemolytic disease of the newborn: a review. Inter. J. of Pharmacotherapy 2015; 5(1): 1-10

16. Ree IMC, Smits-Wintjens VEHJ, Van der Bom JG, Van Klink JMM, Oepkes D, Lopriore E. Neonatal management and outcome in alloimmune hemolytic disease. Expert Rev Hematol 2017; 10(7): 607-616. Doi: $10.1080 / 17474086.2017 .1331124$

17. Esan AJ. Hemolytic Disorders of the Newborn, Current Methods of Diagnosis and Treatment: A Review Study. J Hematol Blood Transfus. 2016; 3: 1-18. Doi: 10.24966/ HBTD-2999/100008

18. Li S, Mo C, Huang L, et al. Hemolytic disease of the fetus and newborn due to alloanti-M: three Chinese case reports and a review of the literature. Transfusion. 2019;59(1):385-395. Doi:10.1111/trf.15054

19. Mawson AR, Croft AM. Rubella Virus Infection, the Congenital Rubella Syndrome, and the Link to Autism. Int. J. Environ. Res. Public Health 2019: 16: 1-28. Doi:10.3390/ijerph16193543

20. Wang L, Liu H. Pathogenesis of aplastic anemia. Hematology. 2019;24(1):559-566. Doi:10.1080/16078 454.2019.1642548

21. Lotero V. Anemia en nińos Deficiencia de hierro. Fundación Valle de Lili. 2010;(165)

22. Acuńa C, Cuero K, Espitia K, Rojas R, Torres R. Anemia drepanocítica y situación en Colombia: Revisión. Bio. 2018;1(3)

23. Lokeshwar MR, Dalal R, Manglani M, Shah N. Anemia in newborn. Indian J Pediatr. 1998;65(5):651-61. Doi: 10.1007/BF02731037

24. Aher S, Malwatkar K, Kadam S. Neonatal anemia. Semin Fetal Neonatal Med. 2008 Aug;13(4):239-47. Doi: 10.1016/j.siny.2008.02.009

25. Kett JC. Anemia in infancy. Pediatr Rev. 2012 Apr;33(4):186-7. Doi: 10.1542/pir.33-4-186

26. Oski FA, Brugnara C, Nathan DG. Un abordaje diagnóstico del paciente anémico. En: Hematología de la infancia y la nińez de Nathan y Oski, 6th, Nathan DG, Orkin SH, Ginsberg D, Look AT (Eds), WB Saunders, Filadelfia 2003. p.409

27. Bhutani VK, Zipursky A, Blencowe H, et al. Neonatal hyperbilirubinemia and Rhesus disease of the newborn: incidence and impairment estimates for 2010 at regional and global levels. Pediatr Res. 2013;74 :86-100. Doi:10.1038/pr.2013.208
28. Hendrickson JE, Delaney M. Hemolytic Disease of the Fetus and Newborn: Modern Practice and Future Investigations. Transfus Med Rev. 2016;30(4):159-164. Doi: 10.1016/j.tmrv.2016.05.008

29. Fikac L. Neonatal Blood Loss Risks. Crit Care Nurs Q. 2019;42(2):202-204. DOI: 10.1097/ CNQ.0000000000000255

30. Cavlovich FE. Subgaleal hemorrhage in the neonate. J Obstet Gynecol Neonatal Nurs. 1995;24(5):397-404. Doi: 10.1111/j.1552-6909.1995.tb02496.x

31. Akin MA, Coban D, Doganay S, Durak Z, Kurtoglu S. Intrahepatic and adrenal hemorrhage as a rare cause of neonatal anemia. J Perinat Med. 2011; 39(3):353-4. Doi: $10.1515 /$ jpm.2011.018

32. Saroha V, Josephson CD, Patel RM. Epidemiology of Necrotizing Enterocolitis: New Considerations Regarding the Influence of Red Blood Cell Transfusions and Anemia. Clin Perinatol. 2019 Mar;46(1):101-117. Doi: 10.1016/j.clp.2018.09.006

33. Carroll PD, Widness JA. Nonpharmacological, blood conservation techniques for preventing neonatal anemia-effective and promising strategies for reducing transfusion. Semin Perinatol. 2012;36(4):232-43. Doi: 10.1053/j.semperi.2012.04.003

34. Sijó A, Saurez G, Velázquez D, Méndez L, Alfonso A, Vargas. Eficacia y seguridad de la eritropoyetina en la anemia de la prematuridad. Rev Cubana Hematol Inmunol Hemoter. 2013;85(2):202-12

35. Juul SE, Vu PT, Comstock BA, Wadhawan R, Mayock DE, Courtney SE, et al. Preterm Erythropoietin Neuroprotection Trial Consortium. Effect of HighDose Erythropoietin on Blood Transfusions in Extremely Low Gestational Age Neonates: Post Hoc Analysis of a Randomized Clinical Trial. JAMA Pediatr. 2020;174(10):933-943. Doi: 10.1001/ jamapediatrics.2020.2271

36. Vu PT, Ohls RK, Mayock DE, German KR, Comstock BA, Heagerty PJ, Juul SE; PENUT Consortium. Transfusions and neurodevelopmental outcomes in extremely low gestation neonates enrolled in the PENUT Trial: a randomized clinical trial. Pediatr Res. 2021:1-8. Doi: 10.1038/s41390-020-01273-w

37. Heddle NM. Pathophysiology of febrile nonhemolytic transfusion reactions. Curr Opin Hematol. 1999;6(6):4206. Doi: 10.1097/00062752-199911000-00012

38. Aher SM, Ohlsson A. Late erythropoiesis-stimulating agents to prevent red blood cell transfusion in preterm or low birth weight infants. Cochrane Database Syst Rev. 2019;2(2):CD004868. Doi: 10.1002/14651858. CD004868.pub5 
39. Cabrera García L, Ruiz Antorán B, Sancho López A. Eritropoyetina: revisión de sus indicaciones. Inf Ter Sist Nac Salud 2009; 33:3-9

40. Figueras J. Eritropoyetina en neonatología. An Pediatr (Barc). 2010;73(6):301-04

41. Neelakantan S, Widness JA, Schmidt RL, Veng-Pedersen P. Erythropoietin pharmacokinetic/pharmacodynamic analysis suggests higher doses in treating neonatal anemia. Pediatr Int. 2009;51(1):25-32. Doi: 10.1111/j.1442-200X.2008.02648.x

42. E. Álvarez E, Pérez JM Figueras J, Estrany C. Tratamiento con eritropoyetina para la anemia tardía tras enfermedad hemolítica del recién nacido. An Pediatr (Barc). 2010; 73(6):334-339

43. Zuppa AA, Alighieri G, Fracchiolla A, Catenazzi P, D'Antuono A, Riccardi R, Cavani M, Romagnoli1 C. Confronto tra due protocolli con Eritropoietina Umana Ricombinante (rHuEpo) nel trattamento dell'anemia tardiva nei neonati con Isoimmunizzazione Rh. Ped. Med. Chir. (Med. Surg. Ped.). 2012, 34: 186-191

44. El-Lahony DM, Saleh NY, Habib MS, Shehata MA, El-Hawy MA. The role of recombinant Human erythropoietin in neonatal anemia. Hematol Oncol Stem Cell 2019. Doi: 10.1016/j.hemonc.2019.08.004

45. Kandasamy Y, Kumar P, Hartley L. The effect of erythropoietin on the severity of retinopathy of prematurity. Eye (Lond). 2014; 28(7):814-8. Doi: 10.1038/eye.2014.95

46. Rangarajan V, Juul SE. Erythropoietin: Emerging Role of Erythropoietin in Neonatal Neuroprotection. Pediatric Neurology 51. 2014. 481- 488

47. Ree IMC, Lopriore E. Updates in Neonatal Hematology: Causes, Risk Factors, and Management of Anemia and Thrombocytopenia. Hematol Oncol Clin North Am. 2019;33(3):521-532. Doi: 10.1016/j.hoc.2019.01.013

48. Widness JA. Pathophysiology of Anemia During the Neonatal Period, Including Anemia of Prematurity. Neoreviews. 2008; 9(11): e520. Doi:10.1542/neo.9$11-\mathrm{e} 520$

49. Lokeshwar MR, Dalal R, Manglani M, Shah N. Anemia in newborn. Indian J Pediatr. 1998;65(5):651-661. Doi:10.1007/BF02731037

50. Lubetzky R, Ben-Shaceras S, Mimani F, Dollberg S. Mode of delivery and neonatal hematocrit. Am J Perinatol. 2000;17(3):163-65

51. Mesquita M, Iramain R, Troche Z. Anemia neonatal dentro de las 24 horas de vida: prevalencia y factores perinatales asociados. Pediatr. (Asunción). 2005;32(1):10-15
52. Letterio J, Pateva I, Petrosiute A, Ahuja S. Hematologic and Oncologic Problems in the Fetus and Neonate. Fanaroff and Martin's Neonatal-Perinatal Medicine. 2020; 79:1416-1475

53. Tutschek B, Hechexr L, Somville T, Bender H. Twin to twin transfusion syndrome complicated by spontaneous mid trimester uterine rupture. J Perinatal Med. 2004; 32(1):95-7

54. Johnsen S, Albrechtsen S, Pirhonen J. Twin to twin transfusion, treated with serial amniocenteses. Acta Obstet Gynecol Scand. 2004; 83(4):326-29

55. Cloherty J, Eichenwald E, Stark A, editores. Manual de cuidados neonatales. Barcelona: Masson; 2005

56. Zonnenberg IA, Vermeulen RJ, Rohaan MW, Van Weissenbruch MM, Groenendaal F, de Vries LS. Severe Neonatal Anaemia, MRI Findings and Neurodevelopmental Outcome. Neonatology. 2016; 109(4):282-288. Doi:10.1159/000443320

57. Rocha G, Pereira S, Antunes-Sarmento J, Flôr-de-Lima F, Soares H, Guimarăes H. Early anemia and neonatal morbidity in extremely low birth-weight preterm infants. J Matern Fetal Neonatal Med. 2019; 1-7. Doi:10. 1080/14767058.2019.1689948

58. Stefanovic V. Fetomaternal hemorrhage complicated pregnancy: risks, identification, and management. Curr Opin Obstet Gynecol. 2016; 28(2):86-94. Doi:10.1097/ GC0.0000000000000248

59. Christensen RD, Lambert DK, Baer VL, et al. Severe neonatal anemia from fetomaternal hemorrhage: report from a multihospital health-care system. J Perinatol. 2013; 33(6):429-434. Doi:10.1038/ jp.2012.142

60. Banerjee J, Asamoah FK, Singhvi D, Kwan AW, Morris JK, Aladangady N. Haemoglobin level at birth is associated with short term outcomes and mortality in preterm infants. BMC Med. 2015; 13:16. Doi:10.1186/ s12916-014-0247-6

61. Brugnara C, Platt OS. The erythrocyte and its disorders. (7th ed.) S.H. Orkin, D.G. Nathan, D. Ginsburg, A.T. Look, D.E. Fisher, S.E. Lux (Eds.), Nathan and Oski's hematology of infancy and childhood, vol. 1, Elsevier, Philadelphia. 2009: 36-66

62. Andersen CC, Collins CL. Poor circulation, early brain injury, and the potential role of red cell transfusion in premature newborns. Pediatrics. 2006;117(4):14641466. Doi:10.1542/peds.2005-3197

63. Sann C, Bourgeois J, Stephen A, Putet G. Outcome of 249 premature infants less than 29 weeks gestational age. Arch Pediatr. 2001; 8(3):250-58 